\title{
Efficienza tecnologica e produttiva delle strutture trasfusionali in Italia
}

Dante Cornago, Livio Garattini

\begin{abstract}
In all the developed countries the methods of blood collection and production are relevant subjects for the pharmacoeconomics studies, because of their health and economic implications related to the health policies in this area.

The "blood market" rappresents an area where the sanitary items, the economic items and also the ethic items must be broached at the same time; therefore it can be considered emblematic.

By this situation arises Progetto CO.R.SA. (COsti Raccolta SAngue), a project for estimating productivity and cost for a representive sample of italian trasfusional structure (ST). The study is supported by Società Italiana di Medicina Trasfusionale e Immunoematologia (SIMTI).
\end{abstract}

In Italy there are about $380 \mathrm{ST}$, but only $30 \mathrm{ST}$ resulted suitable for the study. The analysis investigates six rates concerning:
a) wastes;
b) technological efficiency;
c) personnel productivity.

The present work describes only the informations on ST organization and production, delaying the results about costs to a specific further report.

Farmeconomia e percorsi terapeutici 2002; 3 (1): 28-35

\section{INTRODUZIONE}

Le modalità di raccolta e produzione di sangue ed emoderivati sono soggetto di attualità in tutti i Paesi sviluppati, a causa delle implicazioni sanitarie ed economiche, dirette e indirette (tipicamente gli indennizzi per il danno biologico a trasfusi derivante dalla somministrazione di sangue e suoi derivati) collegate alle scelte di politica sanitaria in tale settore.

Il "mercato del sangue" può essere considerato emblematico, per le sue caratteristiche intrinseche, di quanto sia opportuno affrontare contestualmente gli aspetti sanitari, economici ed etici.

Sotto il profilo economico, data per scontata la necessità di avere a disposizione sangue ed emoderivati il più possibile sicuri (anche per evitare le ripercussioni economiche indirette, sopraccitate, a carico delle autorità pubbliche), sembra auspicabile raggiungere livelli dimensionali di una certa entità nelle strutture trasfusionali, al fine di conseguire economie di scala accettabili a livello "produttivo": analogamente a quanto accade oramai un po' in tutti i settori merceologici, anche nel pur peculiare settore della sanità la ricerca di economie di scala produttive sembra essere ormai una necessità inevitabile.

Vista l'assenza di dati in letteratura che possano in qualche modo fornire una risposta a tale problematica, è emersa l'esigenza di procedere alla stima della produttività e dei costi per tipologia di prestazione su un campione di Strutture Trasfusionali (ST) italiane. Da tale esigenza nasce l'idea del Progetto CO.R.SA. (COsti Raccolta SAngue), condotto con il patrocinio scientifico della Società Italiana di Medicina Trasfusionale e Immunoematologia (SIMTI). Del suddetto progetto, qui verranno presentate solamente le informazioni di natura organizzativa e produttiva, rimandando la presentazione delle stime dei costi a un'ulteriore pubblicazione specifica. 


\section{MERCATODELSANGUE}

In Italia esistono circa $380 \mathrm{ST}$, suddivise fra Servizi di Immunoematologia e Trasfusione (SIT), Centri Trasfusionali (CT) e Unità di Raccolta (UR), ubicate per lo più all'interno delle Aziende Ospedaliere (1); le sole altre istituzioni che possono provvedere alla raccolta di sangue sono la Croce Rossa Italiana e le associazioni di donatori volontari, fra le quali l'AVIS risulta l'unica di rilievo nazionale.

I SIT sono servizi ospedalieri che operano in bacini di utenza superiori a 400.000 abitanti, con una densità minima di uno per provincia. I CT sono strutture ospedaliere che possono essere costituite ad integrazione dei SIT, laddove il bacino di utenza di questi ultimi superino di gran lunga i 400.000 abitanti. Laddove presenti, essi operano in bacini di utenza di almeno 150.000 abitanti e svolgono compiti molto simili a quelli di un SIT. Le UR sono strutture, fisse o mobili, finalizzate alla raccolta di sangue intero e plasma; esse dipendono, sotto il profilo tecnico e organizzativo, dal SIT del territorio di competenza o, laddove presente, dal rispettivo $\mathrm{CT}$.

Il numero delle ST esistenti in Italia è molto più elevato rispetto a quello di altri Paesi europei con popolazione simile, quali Francia e Gran Bretagna (1); tuttavia, va osservato che in Italia sia i SIT che i CT svolgono, oltre all' attività di raccolta propriamente detta, anche attività di Medicina Trasfusionale, svolta in altri servizi ospedalieri nei Paesi europei con minor numero di ST.

Le ST possono decidere di raccogliere il "sangue" al proprio interno oppure di acquistarlo dalle associazioni di donatori sulla base di tariffe nazionali stabilite da un apposito decreto legge (D.L. 5/11/1996). I donatori iscritti alle associazioni di volontari si definiscono "abitudinari", mentre quelli che non aderiscono a nessuna delle suddette associazioni vengono definiti "occasionali", in quanto non garantiscono periodicità e continuità nelle donazioni.

Quando ci si riferisce genericamente al "sangue", si considera sia il sangue intero per separazione (dal quale si ottengono con un processo di lavorazione gli emocomponenti, ossia globuli rossi, piastrine e plasma), sia la raccolta diretta degli emocomponenti tramite un processo "ad hoc" (definito di aferesi).

La separazione degli emocomponenti partendo dal sangue intero si ottiene mediante un processo di stratificazione, a seguito di centrifugazione, collegato al diverso peso specifico delle componenti, utilizzando sacche collegate fra loro in serie da un sistema chiuso; il difetto principale di tale lavorazione è che la precisione non è elevatissima, generando scarti notevoli.
La separazione si rende necessaria sia in virtù del diverso utilizzo clinico delle componenti, sia perché le stesse hanno modalità di conservazione e durata differenti fra loro (ad esempio, le piastrine devono essere separate entro poche ore dal prelievo e conservate, sempre in movimento, per pochi giorni a una temperatura prossima ai $4^{\circ} \mathrm{C}$, mentre i globuli rossi possono essere conservati a $20^{\circ} \mathrm{C}$ per un periodo massimo di circa 40 giorni).

L'aferesi è una modalità di raccolta che, utilizzando lo stesso principio di una centrifuga che fa precipitare le componenti a peso specifico più alto, permette di estrarre solo l'emocomponente che interessa attraverso attrezzature specifiche, rifondendo direttamente nel donatore quelli non richiesti.

Ciascuna ST organizza e gestisce l' attività in modo molto differente, rendendo ogni struttura una realtà pressoché a sé stante; inoltre, la medicina trasfusionale è stata oggetto di un processo di trasformazione e rinnovamento profondo, tuttora in rapida evoluzione, che ha visto il passaggio da una fase di semplice raccolta, conservazione e distribuzione del sangue e degli emoderivati a quella di esecuzione di molte altre procedure. Tutto ciò ha reso necessario un isolamento dell" "attività tipica" delle ST e una classificazione funzionale di tale "core activity" in tre categorie:

1. Raccolta: afferisce a quest' area la sola attività di raccolta sangue operata direttamente dalla ST e finalizzata all'ottenimento di unità di sangue intero, piastrine e plasma da aferesi.

2. Validazione: in quest'area è stata inclusa l'attività di esecuzione dei test diagnostici su tutte le unità di output che li richiedono (cioè tutte le unità di sangue raccolte direttamente dalla ST, più quelle acquistate ancora da validare).

3. Separazione: questa categoria comprende l'attività di separazione di tutto il sangue "intero" (cioè, oltre alle unità raccolte direttamente dalla ST, quelle acquistate), finalizzata principalmente all'ottenimento dei concentrati di globuli rossi, ma anche di piastrine e plasma da separazione.

\section{METODOLOGIA}

Alla selezione del campione di ST da includere nello studio, si è giunti attraverso due fasi consecutive di "campionamento", ciascuna mirata al conseguimento di un risultato propedeutico alla successiva e strumentale al raggiungimento dell' obiettivo finale della ricer- 
ca (cioè la stima dei costi delle ST). La strategia adottata è stata quella di coinvolgere potenzialmente tutte le strutture dotate dei requisiti minimi di partecipazione, definiti in modo sempre più mirato, ma evitando qualsiasi criterio di selezione aprioristica dei partecipanti. In altri termini, è stata concessa l'opportunità a tutte le ST aderenti alla SIMTI di partecipare in modo spontaneo e volontario al progetto.

Inoltre, al fine di poter valutare la significatività del campione in base alla dimensione delle ST, queste ultime sono state raggruppate in tre categorie sulla base del personale complessivamente occupato, espresso in termini di "operatori equivalenti" (cioè di numero di dipendenti "ricalcolati" a tempo pieno):

- strutture piccole (meno di 12 operatori equivalenti);

- $\quad$ strutture medie (fra 12 e 40 operatori equivalenti);

- $\quad$ strutture grandi (più di 40 operatori equivalenti).

Gli intervalli di confidenza per gli indici sono stati calcolati al $95 \%$.

Per poter valutare l'esistenza di correlazioni fra gli indici calcolati (produttività, sprechi e efficienza tecnologica) e la dimensione delle ST misurata in termini di numero di operatori equivalenti, è stata effettuata un' analisi di correlazione semplice su ciascuno di questi indici (2). In particolare, tale analisi è stata condotta senza ricorrere a ulteriori trasformazioni delle variabili, avendo tutti gli indici evidenziato una distribuzione sufficientemente normale.

Le ST sono state raggruppate anche in due categorie a seconda della loro collocazione geografica (Nord e Centro-Sud), per valutare l'esistenza di eventuali differenze statisticamente significative nell' ambito dei suddetti indici; a tale proposito, è stata condotta un' analisi della varianza (ANOVA).

\section{FASE 1}

La prima fase ha previsto l'invio a tutte le 380 ST iscritte alla SIMTI di un questionario "anagrafico" finalizzato a testare la volontà di partecipare al progetto, unitamente alla verifica della presenza del primo requisito formale di partecipazione: la disponibilità di un sistema di controllo di gestione da cui trarre le informazioni necessarie per calcolare successivamente $\mathrm{i}$ costi per tipologia di prestazione.

L'analisi dei 116 questionari restituiti ha portato all'individuazione di 76 strutture dotate dei requisiti di carattere contabile indispensabili ai fini della partecipazione allo studio. Tali strutture sono risultate così distribuite sul territorio nazionale: 43 al nord, 19 al centro e 14 al sud. Le 76 ST sono state riclassificate, in base alla dimensione, in 20 strutture piccole, 48 medie e 8 grandi. Per quanto riguarda la distribuzione dimensionale delle strutture sotto il profilo geografico, è stato rilevato che 7 delle 8 strutture grandi erano localizzate al nord (la struttura rimanente si trovava al sud); delle 48 strutture di medie dimensioni, 26 erano localizzate al nord, 12 al centro e 10 al sud. Infine, le 20 strutture di piccole dimensioni sono risultate così distribuite: 10 al nord, 7 al centro e 3 al sud. Considerando autosufficienti quelle strutture che effettuano un numero di prelievi almeno pari al numero di unità di sangue utilizzate, il $72 \%$ circa delle strutture in analisi si è dichiarato autosufficiente, mentre il restante $28 \%$ ricorre all'acquisto da associazioni di donatori volontari oppure da altre ST.

Essendo stato il campione così ottenuto giudicato rappresentativo della realtà del sistema trasfusionale italiano, è stato inviato un secondo questionario a tutti i responsabili delle 76 strutture ritenute potenzialmente idonee ad accedere alla seconda fase del progetto CO.R.SA.

\section{FASE 2}

La seconda fase è stata mirata alla raccolta di tutte le informazioni di carattere organizzativo e gestionale indispensabili per approfondire $i$ livelli di produttività di ciascuna $\mathrm{ST}$ rispetto ai servizi prestati. In particolare, sono state formulate domande specifiche per comprendere la produttività totale annua della struttura suddivisa per tipologia di prestazione, la destinazione di tutte le unità prodotte (utilizzi, cessioni e eliminazioni per diverse cause), la dotazione di apparecchiature della struttura e il personale ivi impiegato, con la stima dei carichi di lavoro nelle diverse attività svolte.

Dei 76 questionari inviati in questa fase, ne sono pervenuti 45 compilati; di questi, peraltro, solamente 30 hanno risposto in modo completo ed esauriente.

Le 30 strutture selezionate sono risultate così distribuite sul territorio nazionale: 19 al nord, 6 al centro e 5 al sud; sotto il profilo dimensionale, 18 sono risultate piccole, 11 medie e 1 grande.

Per poter procedere nell' analisi statistica, l'unica ST di grandi dimensioni è stata aggregata a quelle medie e le cinque ST del sud sono state compattate con quelle del centro per formare l' aera centro-sud.

Combinando il criterio geografico con quello dimensionale alla luce della riclassificazione di cui sopra, è risultato che, delle 12 strutture me- 
die, 10 sono localizzate al nord e 2 al centrosud, mentre le 18 strutture di piccole dimensioni sono equamente ripartite fra nord e centrosud (Figura 1).

Si è ritenuto che anche il campione emerso dalla seconda fase, seppur geograficamente un po' sbilanciato per quanto riguarda le ST di maggiori dimensioni, potesse ancora essere considerato rappresentativo della realtà del sistema trasfusionale italiano.

L'analisi è consistita nel calcolo di alcuni indici per valutare gli sprechi, l'efficienza tecnologica e la produttività del lavoro. Di seguito, viene riportato sinteticamente il significato di ciascun indice utilizzato nell'analisi e la conseguente interpretazione relativa al suo andamento.

- Indice sprechi: è il rapporto fra il totale degli output eliminati (ponderati per i valori delle relative tariffe) e il totale degli output prodotti (sempre ponderati per i valori delle relative tariffe). Il risultato di tale calcolo è valutato come "proxy" della percentuale degli sprechi sulla produttività totale.

- Indice $A_{1}$ di efficienza tecnologica: è il rapporto fra il numero di sacche di sangue intero separate e il numero di centrifughe utilizzate all'interno della $\mathrm{ST}$; tanto più alto risulta tale valore, migliore dovrebbe essere l'efficienza della struttura, in quanto indicatore di utilizzo più intensivo di ciascuna apparecchiatura.

- Indice $\mathrm{A}_{2}$ di efficienza tecnologica: è il rapporto fra il numero di unità di aferesi raccolte direttamente dalla struttura e il numero di apparecchiature per aferesi utilizzate nei prelievi; anche in questo caso, un valore elevato dell'indice mostra un maggiore numero di prelievi per apparecchiatura e, quindi,

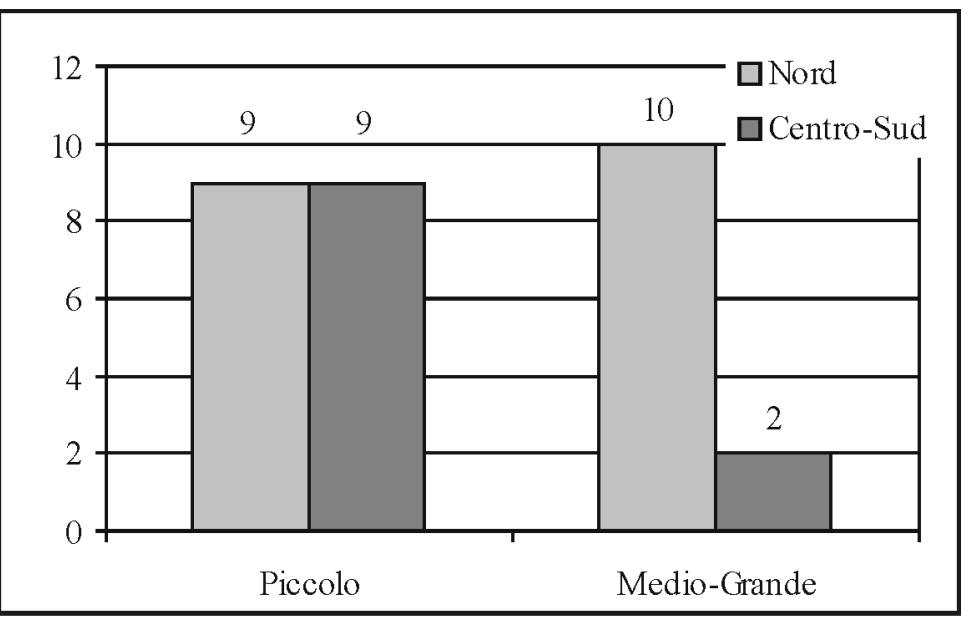

Figura 1

Distribuzione dimensionale del compione di ST analizzato per area geografica un'efficienza superiore della struttura stessa.

Indice $B_{1}$ di produttività del personale: è il rapporto fra il tempo totale dedicato dal personale della struttura addetto all' attività di raccolta diretta del sangue (sia per quello intero sia per le aferesi) e il quantitativo complessivo di unità raccolte direttamente dalla ST. In questo caso, però, una maggior efficienza è associata a un valore inferiore, rappresentando tale indicatore il tempo medio impiegato per la lavorazione di una unità di output.

- Indice $B_{2}$ di produttività del personale: è il rapporto fra il tempo totale dedicato dal personale della struttura addetto all' attività di separazione del sangue intero acquisito (sia quello raccolto direttamente, sia quello acquistato ancora da separare) e il quantitativo complessivo di unità separate dalla ST. Anche in questo caso, analogamente all' indicatore precedente, una maggiore efficienza è associata a un valore inferiore.

Indice $B_{3}$ di produttività del personale: è il rapporto fra il tempo totale dedicato dal personale della struttura addetto all' attività di validazione delle unità acquisite non ancora validate (sia per le unità di sangue intero e di aferesi raccolte direttamente dalla ST, sia per quelle acquistate da validare) e il quantitativo complessivo di unità validate dalla ST. Anche in questo caso una maggiore efficienza è associata a un valore inferiore.

\section{RISULTATI}

La Tabella 1 mostra gli indicatori di sprechi, efficienza tecnologica e produttività delle ST, classificate per dimensione e ordine di grandezza in termini di efficienza crescente.

La media dell'indice degli sprechi è risultata pari a $3,9 \%$, con un intervallo di confidenza pari a 3,1\%-4,8\%. Il volume degli sprechi varia in modo sensibile fra le varie ST, attestandosi solo in cinque casi sotto il $2 \%$. L'analisi statistica non ha mostrato alcuna correlazione significativa fra il volume degli sprechi e la dimensione della ST $\left(\mathrm{R}^{2}=0,007\right.$ con $\left.\mathrm{p}<0,05\right)$. Tale indipendenza appare abbastanza logica, visto che gli sprechi dovrebbero essere collegati, più che alla produttività, a cause tecniche (rottura di sacche, guasti ai sistemi di conservazione, ecc.) e sanitarie (ad esempio, il non superamento dei test obbligatori per legge) o logistiche (mancato utilizzo prima della scadenza). In altri termini, più che alla produttività, gli sprechi dovrebbero essere legati alla gestione e organiz- 


\begin{tabular}{|c|c|c|c|c|c|c|c|}
\hline Dimensione & $\begin{array}{c}\text { Area } \\
\text { geografica }\end{array}$ & $\begin{array}{c}\text { Indice } \\
\text { sprechi } \\
\text { (n. indice) }\end{array}$ & $\begin{array}{c}\text { Indice di } \\
\text { inefficienza } \\
\text { tecnica } \\
\text { (n. indice) }\end{array}$ & $\begin{array}{c}\text { Indice } A_{2} \\
\text { efficienza } \\
\text { tecnica } \\
\text { (n. indice) }\end{array}$ & $\begin{array}{c}\text { Indice } \mathbf{B}_{1} \\
\text { produttività } \\
\text { (n. indice) }\end{array}$ & $\begin{array}{c}\text { Indice } \mathbf{B}_{2} \\
\text { produttività } \\
\text { (n. indice) }\end{array}$ & $\begin{array}{c}\text { Indice }_{B_{3}} \\
\text { produttività } \\
\text { (n. indice) }\end{array}$ \\
\hline $\mathrm{P}$ & C-S & 263,2 & 37,7 & 9,6 & 186,6 & 204,9 & 187,0 \\
\hline $\mathrm{P}$ & C-S & 214,0 & 40,5 & 13,9 & 157,5 & 170,9 & 183,8 \\
\hline $\mathrm{P}$ & $\mathrm{N}$ & 136,7 & 48,2 & 38,4 & 94,7 & 131,8 & 162,5 \\
\hline $\mathrm{P}$ & C-S & 135,3 & 51,1 & 44,9 & 93,6 & 131,6 & 146,3 \\
\hline $\mathrm{P}$ & C-S & 131,3 & 51,1 & 53,1 & 90,5 & 122,7 & 120,8 \\
\hline $\mathrm{P}$ & $\mathrm{N}$ & 114,9 & 52,2 & 53,9 & 82,2 & 78,4 & 102,5 \\
\hline $\mathrm{P}$ & C-S & 105,0 & 60,3 & 55,7 & 76,4 & 70,7 & 96,1 \\
\hline $\mathrm{P}$ & C-S & 101,8 & 60,9 & 60,3 & 76,3 & 62,5 & 67,3 \\
\hline $\mathrm{P}$ & C-S & 93,6 & 62,0 & 65,3 & 74,0 & 61,8 & 60,0 \\
\hline $\mathrm{P}$ & $\mathrm{N}$ & 69,3 & 62,1 & 71,4 & 71,1 & 57,4 & 56,4 \\
\hline $\mathrm{P}$ & C-S & 67,9 & 78,9 & 78,9 & 66,5 & 54,9 & 56,4 \\
\hline $\mathrm{P}$ & $\mathrm{N}$ & 67,6 & 102,2 & 92,4 & 65,4 & 54,1 & 48,4 \\
\hline $\mathrm{P}$ & $\mathrm{N}$ & 64,9 & 104,3 & 97,4 & 65,0 & 51,9 & 47,3 \\
\hline $\mathrm{P}$ & $\mathrm{N}$ & 61,5 & 105,7 & 114,3 & 59,0 & 51,0 & 45,3 \\
\hline $\mathrm{P}$ & $\mathrm{N}$ & 49,0 & 130,4 & 126,8 & 57,5 & 43,5 & 44,7 \\
\hline $\mathrm{P}$ & C-S & 39,6 & 140,7 & 128,4 & 43,2 & 42,7 & 44,6 \\
\hline $\mathrm{P}$ & $\mathrm{N}$ & 31,2 & 180,8 & 143,6 & 42,9 & 21,0 & 31,8 \\
\hline $\mathrm{P}$ & $\mathrm{N}$ & 25,1 & 188,9 & 151,7 & 35,7 & 13,9 & 25,0 \\
\hline Media $P$ & & 77,8 & 98,4 & 86,6 & 79,9 & 79,2 & 84,8 \\
\hline $\mathrm{M}-\mathrm{G}$ & $\mathrm{N}$ & 251,6 & 35,3 & 3,6 & 343,5 & 683,9 & 418,1 \\
\hline $\mathrm{M}-\mathrm{G}$ & C-S & 186,2 & 63,7 & 16,5 & 320,6 & 320,3 & 234,4 \\
\hline M-G & $\mathrm{N}$ & 149,4 & 73,4 & 47,0 & 166,4 & 97,9 & 145,7 \\
\hline M-G & $\mathrm{N}$ & 118,7 & 81,6 & 51,5 & 109,0 & 70,2 & 107,0 \\
\hline $\mathrm{M}-\mathrm{G}$ & $\mathrm{N}$ & 103,8 & 98,0 & 56,1 & 101,3 & 68,8 & 102,3 \\
\hline $\mathrm{M}-\mathrm{G}$ & $\mathrm{N}$ & 77,7 & 106,9 & 61,6 & 89,8 & 64,3 & 99,6 \\
\hline $\mathrm{M}-\mathrm{G}$ & $\mathrm{N}$ & 69,6 & 136,5 & 87,4 & 82,2 & 63,6 & 87,0 \\
\hline M-G & C-S & 69,2 & 147,1 & 91,7 & 80,1 & 62,5 & 77,4 \\
\hline M-G & $\mathrm{N}$ & 65,6 & 150,5 & 95,6 & 74,6 & 52,8 & 76,1 \\
\hline M-G & $\mathrm{N}$ & 55,0 & 159,0 & 108,7 & 68,4 & 32,6 & 54,3 \\
\hline$M-G$ & $\mathrm{~N}$ & 53,2 & 192,1 & 127,2 & 67,6 & 30,2 & 38,4 \\
\hline $\mathrm{M}-\mathrm{G}$ & $\mathrm{N}$ & 28,1 & 197,9 & 853,1 & 58,5 & 27,4 & 33,4 \\
\hline Media $M-G$ & & 133,3 & 102,3 & 120,2 & 130,2 & 131,2 & 122,8 \\
\hline Media & & 100 & 100 & 100 & 100 & 100 & 100 \\
\hline \multicolumn{2}{|c|}{ Intervallo di confidenza } & $77,8-122,2$ & $81,8-118,2$ & $47,3-152,8$ & $74,3-125,7$ & $54,6-145,4$ & $71,4-128,6$ \\
\hline
\end{tabular}

\section{Tabella 1}

Indicatori di sprechi, efficienza tecnica e produttività delle $S T$ classificate per dimensione e ordine di grandezza in termini di efficienza crescente 
zazione del processo di raccolta, conservazione e utilizzo delle unità di sangue.

L'indice $\mathrm{A}_{1}$ di efficienza tecnologica, calcolato sulle centrifughe per la separazione del sangue intero, è mediamente pari a 3425,8 , con un intervallo di confidenza pari a 2802,6-4049. L'ampiezza dell' intervallo denota una notevole variabilità di resa in termini di numero di sacche centrifugate per apparecchiatura all'interno delle varie ST. Va sottolineato come 7 strutture "piccole" del campione siano quelle che hanno registrato un valore più contenuto (cioè una peggiore efficienza tecnologica), mentre 2 strutture "grandi" abbiano registrato i valori più elevati. Tali valori sembrerebbero indicare un legame direttamente proporzionale fra efficienza e dimensione della ST per quanto concerne il volume di sacche di sangue intero trattate, oppure, specularmente, una dotazione eccessiva di apparecchiature rispetto ai bisogni effettivi nelle strutture piccole. Peraltro, il valore dell'indice di correlazione conferma solo parzialmente tale ipotesi $\left(R^{2}=0,27\right.$ con $\left.p<0,05\right)$.

Per quanto concerne l'indice $\mathrm{A}_{2}$ di efficienza tecnologica, calcolato sul numero delle aferesi eseguite all'interno di ciascuna struttura, il valore medio registrato è pari a 449,7 , con un intervallo di confidenza 212,6-687,3. L'ampiezza di tale intervallo testimonia come le diverse strutture gestiscano in modo molto eterogeneo le aferesi. Se da un lato, comunque, la ST più efficiente è una struttura di grandi dimensioni (la stessa dell'indice precedente), va sottolineato come il secondo miglior risultato, ancorché molto distante dal primo (682 contro $3836,5)$, venga registrato da una struttura di piccole dimensioni. Ciononostante, l'analisi statistica dimostra una buona correlazione diretta $\left(\mathrm{R}^{2}=0,60\right.$ con $\left.\mathrm{p}<0,05\right)$ fra la dimensione della $\mathrm{ST}$ e l'efficienza nell'esecuzione delle aferesi.

Una prima analisi degli indici relativi alla produttività del personale $\left(\mathrm{B}_{1}, \mathrm{~B}_{2}\right.$ e $\left.\mathrm{B}_{3}\right)$ conferma come, in pratica, la fase di lavoro che assorbe il maggior periodo di tempo sia (verosimilmente) la raccolta, seguita dalla separazione e dalla validazione. Infatti, 1 'indice $\mathrm{B}_{1}$ della produttività del personale nella raccolta di sangue registra una media pari a 1,33 ore, mentre gli indici $\mathrm{B}_{2}$ (produttività del personale nella separazione) e $\mathrm{B}_{3}$ (produttività del personale nella validazione) hanno medie considerevolmente più contenute (pari, rispettivamente, a $0,41 \mathrm{e}$ 0,19 ore). L'analisi degli intervalli di confidenza mostra come la maggiore variabilità sia registrata dall'indice $\mathrm{B}_{2}(0,10-0,27)$, seguito da $\mathrm{B}_{1}$ e $\mathrm{B}_{3}$ (rispettivamente 0,99-1,67 e 0,29-0,53). Contrariamente alle aspettative, l'analisi statistica di questi indici di produttività del personale non ha dimostrato alcuna correlazione significativa con il numero degli addetti, risultando i valori di $R_{2}$ del tutto trascurabili (rispettivamente,
$\mathrm{R}^{2}=0.0015, \mathrm{R}^{2}=0.0001$ e $\mathrm{R}^{2}=0.0022$ con $\left.\mathrm{p}>0.05\right)$.

Infine, gli indici sono stati raggruppati in due gruppi per area geografica (Nord e CentroSud), al fine di valutare l'esistenza di eventuali differenze significative rispetto all'ubicazione geografica della struttura. L'analisi della varianza (ANOVA) non ha mostrato alcun risultato significativo.

\section{DISCUSSIONE}

Il limite principale del presente studio riguarda l'utilizzo di dati provenienti da questionari compilati direttamente dalle ST. E', infatti, possibile supporre che l'attendibilità di molti dati possa essere stata inficiata da fattori vari, quali ad esempio, la fretta nella compilazione del questionario e l'indisponibilità di informazioni disaggregate all'interno della ST. Peraltro, per cercare di limitare a priori il rischio di raccogliere dati prevedibilmente inattendibili, si è cercato di predisporre questionari il più possibile semplificati, ideati in collaborazione con operatori del settore, la cui compilazione richiedesse informazioni verosimilmente reperibili all'interno delle strutture.

Dall' analisi è emerso che gli unici indici in qualche misura correlati con la dimensione della struttura sono risultati quelli relativi all'efficienza tecnologica, con particolare riferimento alle attrezzature per aferesi. Se, da un lato, è ragionevole non attendersi correlazioni particolari fra il livello degli sprechi e la dimensione della ST, va d'altro canto sottolineato come sarebbe stato ragionevole ipotizzare una correlazione significativa fra la dimensione della ST e la produttività del personale (relativamente sia alla fase di raccolta, validazione o separazione). Tuttavia, il risultato dell' analisi statistica non supporta l'assunto secondo il quale una struttura di maggiori dimensioni (cioè con un numero maggiore di addetti) dovrebbe raggiungere livelli di efficienza superiori, consentendo quindi una maggiore intensità produttiva e conseguenti economie di scala. Tale risultato potrebbe essere giustificato, soprattutto a causa dell' attività di raccolta, dal fatto che il settore è caratterizzato da una domanda di breve periodo di natura casuale che potrebbe non consentire una precisa programmazione, a scapito dell'efficienza produttiva (3). Inoltre, le inefficienze produttive potrebbero essere riconducibili anche a una scarsa sincronizzazione delle varie fasi produttive, unitamente a un'elevata eterogeneità a livello locale delle modalità di gestione delle attività collaterali alla lavorazione (gestione donatori, refertazione esami controllo, segreteria, amministrazione ecc.). Infine, alla luce dei noti condizionamenti politici che hanno caratterizzato l'evoluzione storica del SSN, 
nonché dell'assenza di responsabilizzazione terminata in modo sostanzialmente avulso dal economica da parte dei livelli locali dello stes- fabbisogno reale. Studi ulteriori ad hoc sarebso fino a tempi recentissimi, non è possibile bero necessari per verificare quale delle ipotesi nemmeno scartare a priori l'ipotesi che la dota- formulate potrebbe essere più confacente a rapzione di personale delle ST possa essersi de- presentare la realtà italiana.

\section{BIBLIOGRAFIA}

1. Istituto Superiore di Sanità 1995. Mappa delle strutture trasfusionali esistenti sul territorio nazionale.

2. Scardovi I, Monari P. Metodologia statistica in biologia e medicina. Bologna: Utet, 1991.

3. Van Der Pol M M, Cairins J A. The efficient organization of blood donation. Health Economics 7: 455-463 (1998). 